\title{
Risk Managing Bermudan Swaptions in the Libor BGM Model ${ }^{1}$
}

\author{
Raoul Pietersz ${ }^{2}$, Antoon Pelsser ${ }^{3}$
}

Econometric Institute Report EI 2003-33

17 August 2003

\begin{abstract}
This article presents a novel approach for calculating swap vega per bucket in the Libor BGM model. We show that for some forms of the volatility an approach based on re-calibration may lead to a large uncertainty in estimated swap vega, as the instantaneous volatility structure may be distorted by re-calibration. This does not happen in the case of constant swap rate volatility. We then derive an alternative approach, not based on re-calibration, by comparison with the swap market model. The strength of the method is that it accurately estimates vegas for any volatility function and at a low number of simulation paths. The key to the method is that the perturbation in the Libor volatility is distributed in a clear, stable and well understood fashion, whereas in the re-calibration method the change in volatility is hidden and potentially unstable.
\end{abstract}

Key words: central interest rate model, Libor BGM model, swaption vega, risk management, swap market model, Bermudan swaption

\section{JEL Classification: G13}

\footnotetext{
${ }^{1}$ We are grateful for the comments of Steffan Berridge, Nam Kyoo Boots, Dick Boswinkel, Igor Grubišić, Les Gulko, Karel in 't Hout, Etienne de Klerk, Steffen Lukas, Mike Monoyios, Maurizio Pratelli, Marcel van Regenmortel, Kees Roos and seminar participants at ABN AMRO Bank, Blaise Pascal International Conference on Financial Modelling Paris, Delft University of Technology, Global Finance Conference Frankfurt/Main and Tilburg University. All errors are our own.

${ }^{2}$ Erasmus Research Institute of Management, Erasmus University Rotterdam, P.O. Box 1738, 3000 DR Rotterdam, The Netherlands (email: pietersz@few.eur.nl, tel: +31 10 4088932, fax: +31 10 4089136) and Market Risk - Modelling and Product Analysis (HQ1059), ABN AMRO Bank, P.O. Box 283, 1000 EA Amsterdam, The Netherlands

${ }^{3}$ Econometric Institute, Faculty of Economics, Erasmus University Rotterdam, P.O. Box 1738, 3000 DR Rotterdam, The Netherlands (email: pelsser@few.eur.nl, tel: +31 10 4081270, fax: +31 10 4089169) and Nationale-Nederlanden, P.O. Box 796, 3000 AT Rotterdam, The Netherlands
} 


\section{Introduction}

The Libor BGM interest rate model was introduced by Brace, Gạtarek, Musiela [BGM97], Jamshidian [Jam96] [Jam97] and by Miltersen, Sandmann, Sondermann [MSS97]. This model is presently most popular amongst both academics and practitioners alike. The reasons for its popularity are numerous, but possibly most important is that the Libor BGM model has the potential for risk managing exotic interest rate derivatives that depend on both the cap and swaption markets. In other words, BGM has the potential of becoming the central interest rate model. It features log-normal Libor rates and almost log-normal swap rates and consequently also the market standard Black formula for caps and swaptions. Approximate swaption volatility formulas exist in the literature (e.g. [HuW00]) and have been shown to be of high quality (e.g. [BDB98]).

There are however still a number of issues that need to be resolved to achieve the goal of using BGM as the central interest rate model. One of these issues is the calculation of swap vega. A common and usually very successful method for calculating a Greek in a model equipped with a calibration algorithm is to perturb market input, re-calibrate and then re-value the option. The difference in value divided by the perturbation size is then an estimate for the Greek. If however this technique is applied to the calculation of swap vega in the Libor BGM model, then it may (depending on the volatility function) yield estimates with large uncertainty. In other words, the standard error of the vega is relatively high. The uncertainty disappears of course by increasing the number of simulation paths, but the number required for clarity can by far supersede 10,000, which is probably the maximum in a practical environment. This large uncertainty in vega has been illustrated in section 2 , most notably in figure 2 .

For a constant volatility calibration however the vega is estimated with low uncertainty. The number of simulation paths needed for clarity of vega thus depends on the chosen calibration. The cause is that for certain calibrations, under a perturbation, the additional volatility is distributed non-evenly and, may one even say, unstably over time. For the constant volatility calibration of course this additional volatility is naturally distributed evenly over time. It follows that the correlation between the discounted payoff along the original and perturbed volatility is larger. As the vega is the expectation of the difference between these payoffs (divided by the perturbation size) consequently the standard error will be lower.

A method is developed, not based on re-calibration, for computing swap vega per bucket in the Libor BGM model. It may be used to calculate swap vega in the presence of any volatility function, with clarity already for 10,000 
simulation paths or less. The strength of the method is that it accurately estimates swap vegas for any volatility function and at a low number of simulation paths. The key to the method is that the perturbation in the Libor volatility is distributed in a clear, stable and well understood fashion, whereas in the re-calibration method the change in volatility is hidden and potentially unstable. The method is based on keeping swap rate correlation fixed while increasing the swap rate instantaneous volatility evenly over time, for only a single swap rate; all other swap rate volatility remains unaltered.

It is important to verify that a calculation method reproduces the correct numbers in a situation where the answer is known. We choose to benchmark our swap vega calculation method using Bermudan swaptions. There are two reasons for this: First, a Bermudan swaption is a complicated enough (swap-based) product (in a Libor-based model) that depends non-trivially on the swap rate volatility dynamics; for example, its value depends also on swap rate correlation. Second, a Bermudan swaption is not as complicated as certain other more exotic interest rate derivatives and some intuition exists about its vega behaviour. We show for Bermudan swaptions that our method yields similar swap vega as found in a swap market model.

Finally, we mention the paper of Glasserman and Zhao [GlZ99], who provide efficient algorithms for calculating risk sensitivities given a perturbation of Libor volatility. Our problem differs from theirs in that we derive a method to calculate the perturbation of Libor volatility to obtain the correct swap rate volatility perturbation for swaption vega. The Glasserman and Zhao approach may then be applied to efficiently compute the swaption vega given the Libor volatility perturbation found by our method.

The remainder of this paper is organized as follows. First, we present the approach to calculating swap vega per bucket based on re-calibration with a specific volatility function. We show that resulting swap vega may be poorly estimated at a low number of simulation paths. Second, the natural definition of swap vega in the canonical swap market model (SMM) is studied. Third, the SMM-definition of swap vega is extended to Libor BGM. Fourth, we return to the re-calibration approach and using the previously developed theory we are able to explain the poorly estimated vega. Fifth, correct numerical swap vega results for a 30 year deal are presented. Sixth, we show that similar swap vega are obtained in a swap market model. The article ends with conclusions.

\section{Re-calibration Approach}

In this section we consider examples of the re-calibration approach of computing swap vega. Three calibration methods are considered. It is shown 
that, for two of the three methods, resulting vega is hard to estimate, i.e., a large number of simulation paths is needed for clarity. To facilitate our discussion, first some notation is introduced.

BGM. Consider a BGM model. Such a model features a tenor structure $0<T_{1}<\cdots<T_{N+1}$ and $N$ forward rates $L_{i}$ accruing from $T_{i}$ to $T_{i+1}$, $i=1, \ldots, N$. Each forward rate is modelled as a geometric Brownian motion under its forward measure,

$$
\frac{\mathrm{d} L_{i}(t)}{L_{i}(t)}=\bar{\sigma}_{i}(t) \cdot \mathrm{d} \bar{W}^{i+1}(t), \quad 0 \leq t \leq T_{i},
$$

Here $\bar{W}^{i+1}$ is a $d$-dimensional Brownian motion under the forward measure $\mathbb{Q}_{i+1}$. The positive integer $d$ is referred to as the number of factors of the model. We consider a full-factor model so we set $d$ equal to $N$. The function $\bar{\sigma}_{i}:\left[0, T_{i}\right] \rightarrow \mathbb{R}^{d}$ is the volatility vector function of the $i^{\text {th }}$ forward rate. The $k^{\text {th }}$ component of this vector corresponds to the $k^{\text {th }}$ Wiener factor of the Brownian motion.

A discount bond pays one unit of currency at maturity. The time-t price of a discount bond with maturity $T_{i}$ is denoted by $B_{i}(t)$. The forward rates are related to discount bond prices as follows

$$
L_{i}(t)=\frac{1}{\delta_{i}}\left\{\frac{B_{i}(t)}{B_{i+1}(t)}-1\right\} .
$$

Here $\delta_{i}$ is the accrual factor for the time span $\left[T_{i}, T_{i+1}\right]$.

Swap Rates and BGM. The swap rate corresponding to a swap starting at $T_{i}$ and ending at $T_{j+1}$ is denoted by $S_{i: j}$. The swap rate is related to discount bond prices as follows

$$
S_{i: j}(t)=\frac{B_{i}(t)-B_{j+1}(t)}{\operatorname{PVBP}_{i: j}(t)} .
$$

Here PVBP denotes the principal value of a basis point,

$$
\operatorname{PVBP}_{i: j}(t)=\sum_{k=i}^{j} \delta_{k} B_{k+1}(t) .
$$

It is understood that $\mathrm{PVBP}_{i: j} \equiv 0$ whenever $j<i$. We will consider the swap rates $S_{1: N}, \ldots, S_{N: N}$ corresponding to the swaps underlying a co-terminal Bermudan swaption ${ }^{4}$. Swap rate $S_{i: N}$ is a martingale under its forward swap

\footnotetext{
${ }^{4} \mathrm{~A}$ co-terminal Bermudan swaption is an option to enter into an underlying swap at several exercise opportunities. In other words the holder of a Bermudan swaption has the right at each exercise opportunity to either enter into a swap or hold on to the option; all underlying swaps that may possibly be entered into share the same end date.
} 
measure $\mathbb{Q}_{i: N}$. We may thus implicitly define its volatility vector $\bar{\sigma}_{i: N}$ by

$$
\frac{\mathrm{d} S_{i: N}(t)}{S_{i: N}(t)}=\bar{\sigma}_{i: N}(t) \cdot \mathrm{d} \bar{W}^{i: N}(t), \quad 0 \leq t \leq T_{i}
$$

In general $\bar{\sigma}_{i: N}$ will be stochastic because swap rates are not log-normally distributed in the BGM model. These are however distributed very close to log-normal as shown for example by [BDB98]. Because of near log-normality the Black formula approximately holds for European swaptions. Closed-form formulas exist in the literature for the swaption Black implied volatility, see for example $[\mathrm{HuW00]}$. The swap rate volatility formula will be treated in more detail in section 4 .

We choose to model the Libor instantaneous volatility as constant in between tenor dates (piece-wise constant).

Definition 1 (Piece-wise constant volatility) A volatility structure $\left\{\bar{\sigma}_{i}(\cdot)\right\}_{i=1}^{N}$ is piece-wise constant if

$$
\bar{\sigma}_{i}(t)=(\text { const }), \quad t \in\left[T_{i-1}, T_{i}\right) .
$$

The volatility will sometimes be modelled as time homogeneous.

Definition 2 (Time homogeneity) A fixing is defined to be one of the time points $T_{1}, \ldots, T_{N}$. Define $\iota:[0, T] \rightarrow\{1, \ldots, N\}$,

$$
\iota(t)=\#\{\text { fixings in }[0, t)\} .
$$

A volatility structure is said to be time homogeneous if it depends only on the index to maturity $i-\iota(t)$.

Calibrations. Three volatility calibration methods are considered:

1 (THFRV) Time homogeneous forward rate volatility. This approach is based on ideas in [Reb01]. Because of the time homogeneity restriction, there remain as many parameters as market swaption volatilities. A Newton Rhapson type solver may be used to find the exact calibration solution (if such exists).

2 (THSRV) Time homogeneous swap rate volatility. The algorithm for calibrating with such volatility function is a two stage bootstrap, as outlined in appendix A.

3 (CONST) Constant forward rate volatility. Note that constant forward rate volatility implies constant swap rate volatility. The corresponding calibration algorithm is similar to the second stage of the two stage bootstrap. 
Table 1: Market European swaption volatilities.

\begin{tabular}{rccccccc}
\hline Expiry (Y) & 1 & 2 & 3 & $\ldots$ & 28 & 29 & 30 \\
Tenor (Y) & 30 & 29 & 28 & $\ldots$ & 3 & 2 & 1 \\
\hline $\begin{array}{r}\text { Swaption } \\
\text { Volatility }\end{array}$ & $15.0 \%$ & $15.2 \%$ & $15.4 \%$ & $\ldots$ & $20.4 \%$ & $20.6 \%$ & $20.8 \%$ \\
\hline
\end{tabular}

All calibration methods have in common that the forward rate correlation structure is calibrated to a historic correlation matrix via principal components analysis (PCA), see [HuW00]. Correlation is assumed to evolve time homogeneously over time.

Market Data. We considered a 31NC1 co-terminal Bermudan payer's swaption deal struck at $5 \%$ with annual compounding. The notation $x \mathrm{NC} y$ denotes an ' $x$ non-call $y$ ' Bermudan option, which is exercisable into a swap with a maturity of $x$ years and callable only after $y$ years. The option is callable annually. The BGM tenor structure is $0<1<2<\cdots<31$. All forward rates are taken to equal $5 \%$. The time-zero forward rate instantaneous correlation is assumed given by the Rebonato form [Reb98] p. 63,

$$
\rho_{i j}(0)=e^{-\beta\left|T_{i}-T_{j}\right|} .
$$

Here $\beta$ is chosen to equal 0.05 . The market European swaption volatilities were taken as displayed in table 1.

Longstaff Schwartz. To determine the exercise boundary the Longstaff Schwartz least squares Monte Carlo method [LoS01] was used. Only a single explanatory variable was considered, namely the swap net present value (NPV). Two regression functions were employed, a constant and linear term.

Re-Calibration Approach Swap Vega per Bucket. For each bucket a small perturbation $\Delta \sigma\left(\approx 10^{-8}\right)$ was applied to the swaption volatility in the calibration input data ${ }^{5}$. The model was re-calibrated and it was checked that the calibration error for all swaption volatilities was a factor $10^{6}$ smaller than the volatility perturbation. The Bermudan swaption was re-priced through Monte Carlo simulation using the exact same random numbers. Denote the

\footnotetext{
${ }^{5}$ It was verified that the resulting vega is stable for a whole wide range of volatility perturbation sizes. For very extreme perturbation sizes the vega however deviates: If the volatility perturbation size is chosen too large, then vega-gamma terms affect the vega. If the volatility perturbation size is chosen too small, then floating number round-off errors affect the vega.
} 


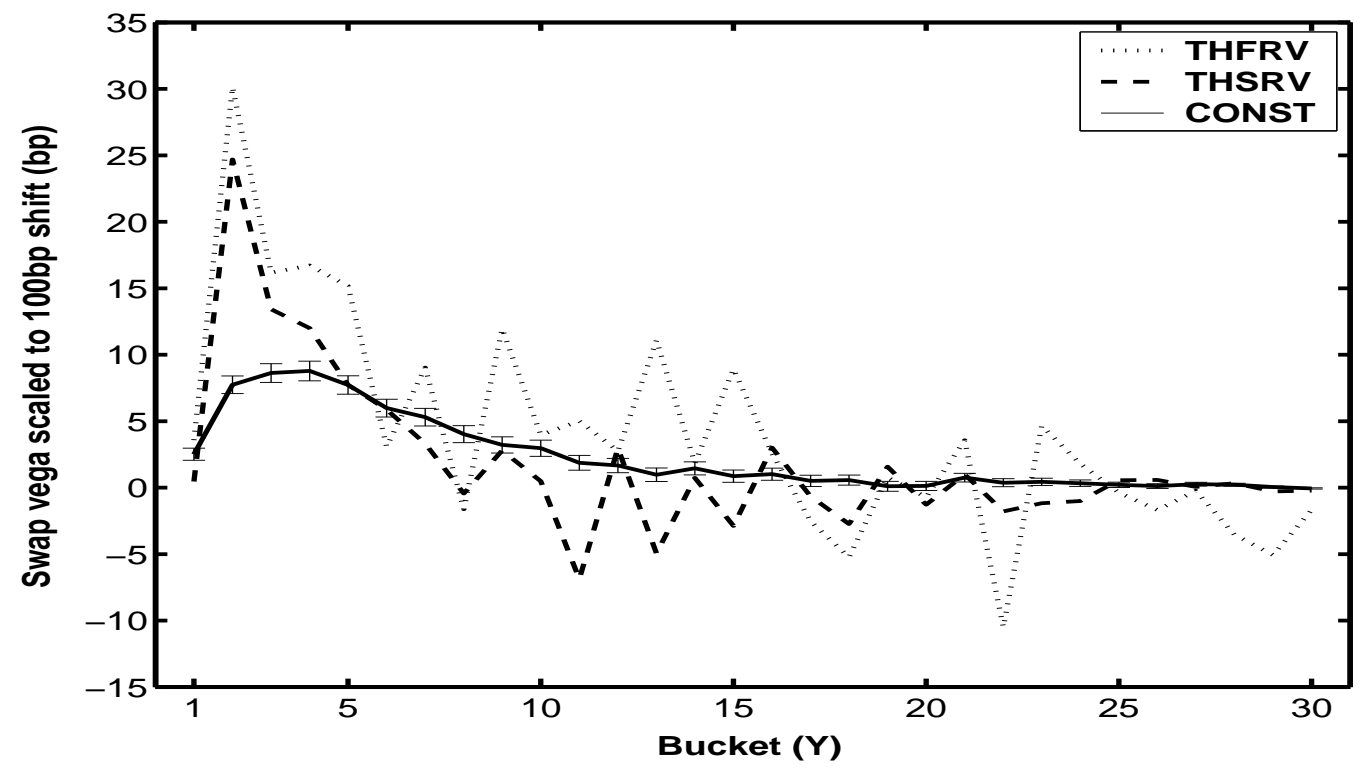

Figure 1: Re-calibration swap vega results for 10,000 simulation paths. Error bars around the CONST vegas denote a $95 \%$ confidence bound based on twice standard error. The vega is a scaled numerical derivative and we verified that it is insensitive to the actual size of the small volatility perturbation used (see also remark 3).

original price by $V$ and the perturbed price by $V_{i: N}$. Then the re-calibration method of estimating swap vega $\mathcal{V}_{i: N}$ for bucket $i$ is given by

$$
\mathcal{V}_{i: N}=\frac{V_{i: N}-V}{\Delta \sigma}
$$

Remark 3 (Scaled Swap Vega) Usually the swap vega is denoted in terms of a shift in the swaption volatility. For example, consider a 100 basis point (bp) shift in the swaption volatility. The swap vega scaled to a $100 \mathrm{bp}$ shift $\mathcal{V}_{i: N}^{100 \mathrm{bp}}$ is then defined by

$$
\mathcal{V}_{i: N}^{100 \mathrm{bp}}=(0.01) \cdot \mathcal{V}_{i: N}
$$

Results. Swap vega results for a Monte Carlo simulation of 10,000 scenarios have been displayed in figure 1. The standard errors have been displayed separately in figure 2. Figure 3 displays the THFRV vega for 1,000,000 simulation paths. The actual vega numbers are more clear, along with a lower standard error. Note however that clarity is obtained already at 10,000 paths for the constant volatility calibration. 


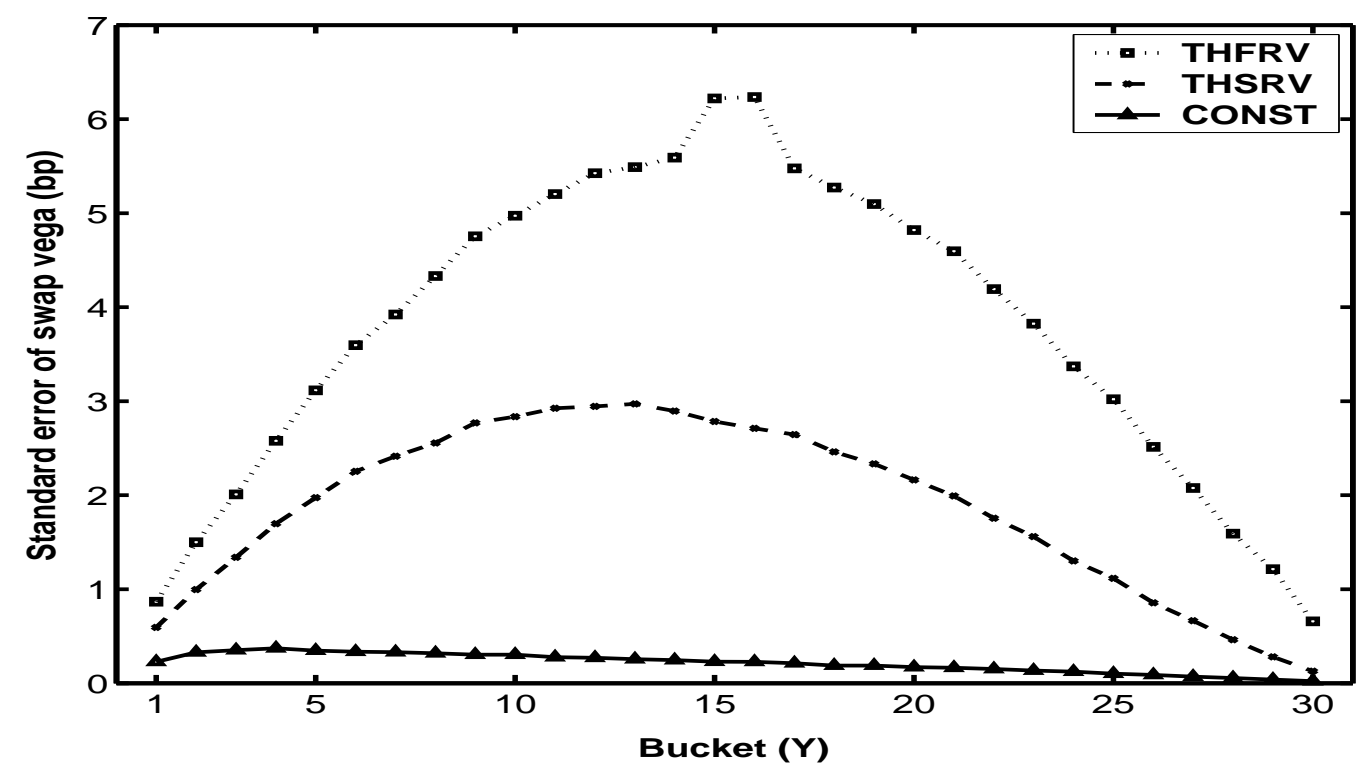

Figure 2: Empirical standard errors of the vega for 10,000 simulation paths.

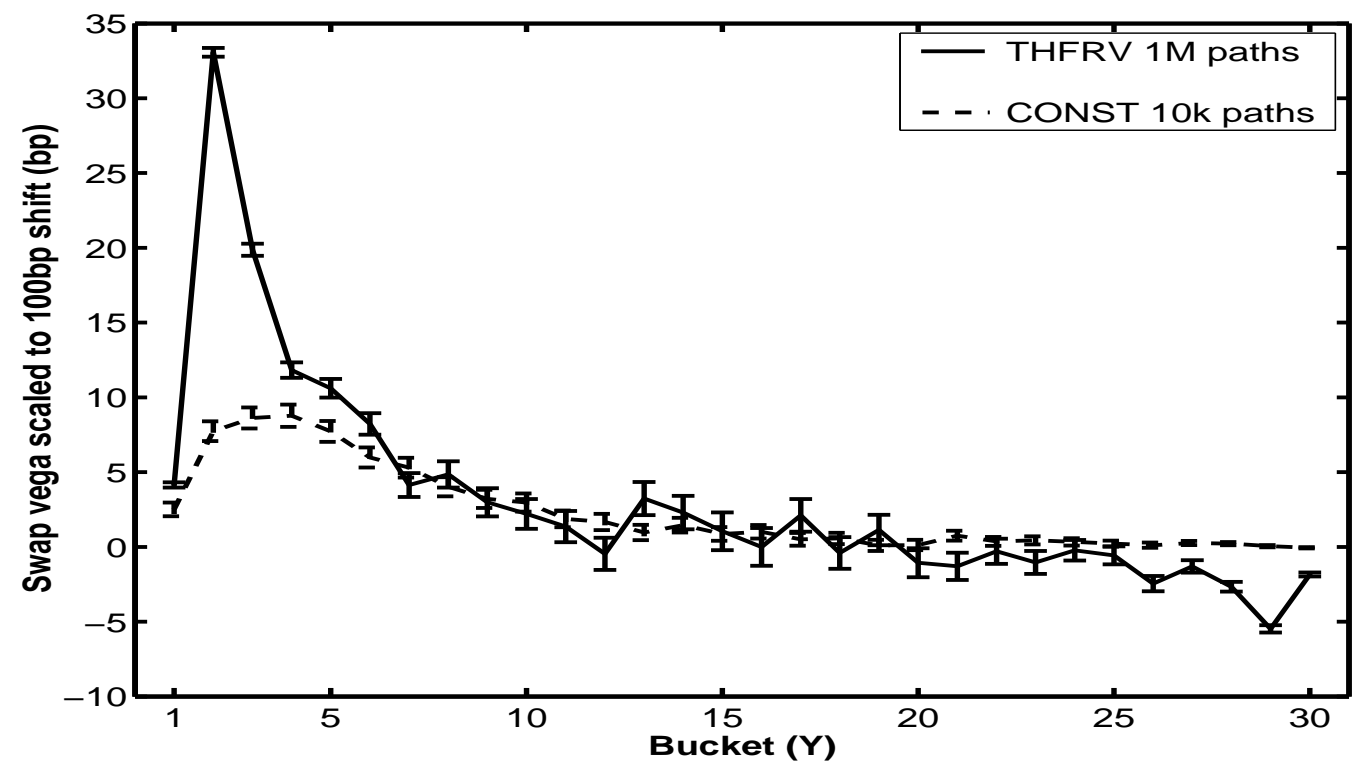

Figure 3: Re-calibration THFRV vega results for 1,000,000 simulation paths. Error bars denote a $95 \%$ confidence bound based on the standard error. The vega is a scaled numerical derivative and we verified that it is insensitive to the actual size of the small volatility perturbation used (see also remark 3). 
Vega Standard Error. The standard error of the swap vega is calculated as follows. A Monte Carlo simulation may be interpreted as a finite discretization of the probability space. If $M$ is the number of scenarios then the finite probability space $\Omega$ is given by the set of scenarios $\left\{\omega_{1}, \ldots, \omega_{M}\right\}$ with probability measure $\mathbb{P}$ determined by $\mathbb{P}\left(\omega_{i}\right)=1 / M$ for all $i$. Let $P(\omega)$ and $P_{i: N}(\omega)$ denote the discounted payoff of the Bermudan swaption along a scenario $\omega$ for the original volatility scenario and the perturbed volatility scenario, respectively. The scaled swap vega in this discretized world may then be computed through the following expression

$$
\mathcal{V}_{i: N}^{100 \mathrm{bp}}=(0.01) \frac{V_{i: N}-V}{\Delta \sigma_{i: N}}=c \mathbb{E}\left[P_{i: N}-P\right], \quad c:=\frac{(0.01)}{\Delta \sigma_{i: N}} .
$$

The vega standard error is thus proportional to the standard error of the series $\left\{\left(P_{i: N}-P\right)\left(\omega_{i}\right)\right\}_{i=1}^{M}$, with proportionality constant $c$.

\section{Swap Vega and the Swap Market Model}

In this section we study the natural definition of swap vega in the canonical swap market model. This definition will then be extended to Libor BGM, which will help us explain the results of the previous section. Moreover, it will provide us with an alternative method of calculating swap vega per bucket.

How much European swaptions our dynamically managed hedging portfolio should hold is essentially determined by the swap vega per bucket. The latter is the derivative of the exotic price with respect to the Black implied swaption volatility. Consider a swap market model $\mathcal{S}$. In the latter, swap rates are log-normally distributed under their forward swap measure. This means that all swap rate volatility functions $\bar{\sigma}_{i: N}(\cdot)$ of equation (1) are deterministic. The Black implied swaption volatility $\sigma_{k: N}$ is given by

$$
\sigma_{k: N}=\sqrt{\frac{1}{T_{k}} \int_{0}^{T_{k}}\left|\bar{\sigma}_{k: N}(s)\right|^{2} \mathrm{~d} s .}
$$

As may be seen from the above equation, there are an uncountable number of perturbations of the swap rate instantaneous volatility to obtain the very same perturbation of the Black implied swaption volatility. There is however a natural 1-dimensional parameterized perturbation of the swap rate instantaneous volatility, namely a simple proportional increment. This has been illustrated in figure 4.

Definition 4 (Definition of Swap Vega in a Swap Market Model) Denote the price of an interest rate derivative in a swap market model $\mathcal{S}$ by $V$. Consider 


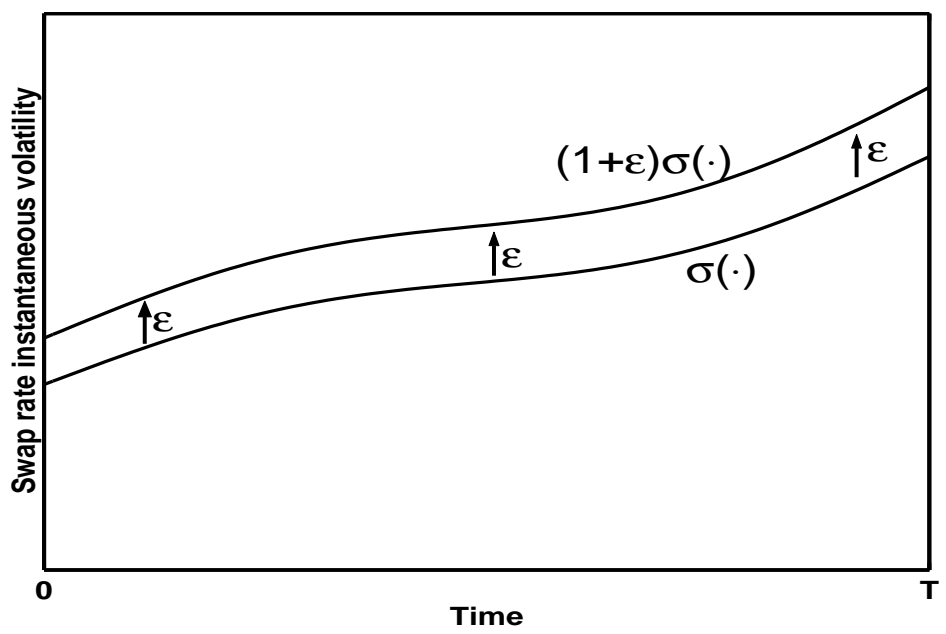

Figure 4: Natural increment of Black implied swaption volatility through proportional increment of swap rate instantaneous volatility: $\sigma(\cdot)$ becomes $(1+\varepsilon) \sigma(\cdot)$.

for all real $\varepsilon$ in an open neighbourhood of 0 a perturbation of the swap rate instantaneous volatility given by

$$
\bar{\sigma}_{k: N}^{\varepsilon}(\cdot)=(1+\varepsilon) \bar{\sigma}_{k: N}(\cdot)
$$

the shift applied only to $k: N$. Denote the corresponding swap market model by $\mathcal{S}_{k: N}(\varepsilon)$. Note that the implied swaption volatility in $\mathcal{S}_{k: N}(\varepsilon)$ is given by $\sigma_{k: N}^{\varepsilon}=(1+\varepsilon) \sigma_{k: N}$. Denote the price of the derivative in $\mathcal{S}_{k: N}(\varepsilon)$ by $V_{k: N}(\varepsilon)$. Then the swap vega per bucket $\mathcal{V}_{k: N}$ is naturally defined as

$$
\mathcal{V}_{k: N}=\lim _{\varepsilon \rightarrow 0} \frac{V_{k: N}(\varepsilon)-V}{\varepsilon \sigma_{k: N}}
$$

Equation (4) is the derivative of the exotic price with respect to the Black implied swaption volatility; using suggestive notation we may write

$$
\mathcal{V}_{k: N}=\frac{\partial V}{\partial \sigma_{k: N}}=\lim _{\Delta \sigma_{k: N} \rightarrow 0} \frac{V\left(\sigma_{k: N}+\Delta \sigma_{k: N}\right)-V\left(\sigma_{k: N}\right)}{\Delta \sigma_{k: N}}
$$

In equation (4) $\varepsilon \sigma_{k: N}$ is equal to the swaption volatility perturbation $\Delta \sigma_{k: N}$ and $V_{k: N}(\varepsilon)$ and $V$ denote the prices of the derivative in models in which the $k^{\text {th }}$ swaption volatility equals $\sigma_{k: N}+\Delta \sigma_{k: N}$ and $\sigma_{k: N}$, respectively. 
Remark 5 (Relative versus absolute shifting) The swap rate volatility perturbation of equation (3) defines a relative shift. It is also possible to apply an absolute shift in the form of

$$
\bar{\sigma}_{k: N}^{\varepsilon}(\cdot)=\left(1+\frac{\varepsilon}{\left|\bar{\sigma}_{k: N}(\cdot)\right|}\right) \bar{\sigma}_{k: N}(\cdot),
$$

the shift applied only to $k: N$. This ensures that the absolute level of the swap rate instantaneous volatility is increased by an amount $\varepsilon$. Note that the relative and absolute perturbation are equivalent when the instantaneous volatility is constant over time. The method for calculating swap vega per bucket is largely the same for both the relative and absolute perturbation. If there are any differences then these will be pointed out in the text. The first difference is in the change in swaption implied volatility $\Delta \sigma_{k: N}$ of equation (5), namely the perturbed volatility satisfies

$$
\begin{aligned}
\sigma_{k: N}^{\varepsilon} & =\sqrt{\frac{1}{T_{k}} \int_{0}^{T_{k}}\left\{\left|\bar{\sigma}_{k: N}(s)\right|+\varepsilon\right\}^{2} \mathrm{~d} s} \\
& =\sqrt{\frac{1}{T_{k}} \int_{0}^{T_{k}}\left|\bar{\sigma}_{k: N}(s)\right|^{2} \mathrm{~d} s+\varepsilon \frac{2}{T_{k}} \int_{0}^{T_{k}}\left|\bar{\sigma}_{k: N}(s)\right| \mathrm{d} s+\mathcal{O}\left(\varepsilon^{2}\right)} \\
& =\sigma_{k: N}+\varepsilon \frac{\frac{1}{T_{k}} \int_{0}^{T_{k}}\left|\bar{\sigma}_{k: N}(s)\right| \mathrm{d} s}{\sigma_{k: N}}+\mathcal{O}\left(\varepsilon^{2}\right) .
\end{aligned}
$$

\section{Alternative Method for Calculating Swap Vega}

In this section an alternative method for calculating swap vega in the BGM framework is presented. It may be applied to any volatility function to yield accurate vega at a low number of simulation paths. The method is based on a perturbation in the forward rate volatility to match a constant swap rate volatility increment. The method is briefly hinted at in section 10.6 .3 of [Reb02] in terms of covariance matrices. In comparison, our derivation below is written more insightfully in terms of the volatility vectors.

Swap rates are not log-normally distributed in Libor BGM. This means that swap rate instantaneous volatility is stochastic. The stochasticity is however almost not apparent as shown empirically for example by [BDB98]. In section 1.5.5 of [D'A02] it is shown that the swap rate is uniformly close to a log-normal martingale.

In $[\mathrm{HuW00}]$ it is shown that the swap rate volatility vector is a weighted 
average of forward Libor rate volatility vectors;

$$
\begin{gathered}
\bar{\sigma}_{i: N}(t)=\sum_{j=1}^{N} w_{j}^{i: N}(t) \bar{\sigma}_{j}(t), \quad w_{j}^{i: N}(t)=\frac{\delta_{j} \gamma_{j}^{i: N}(t) L_{j}(t)}{1+\delta_{j} L_{j}(t)}, \\
\gamma_{j}^{i: N}(t)=\frac{B_{i}(t)}{B_{i}(t)-B_{N+1}(t)}-\frac{\operatorname{PVBP}_{i:(j-1)}(t)}{\operatorname{PVBP}_{i: N}(t)}
\end{gathered}
$$

where the weights $w^{i: N}$ are in general state-dependent.

Hull and White derive an approximating formula for European swaption prices based on evaluating the weights in equation (7) at time zero. The quality of this approximation is high in virtue of the near log-normality of swap rates in Libor BGM. We will denote the resulting swap rate instantaneous volatility by $\bar{\sigma}_{i: N}^{\mathrm{HW}}$, thus

$$
\bar{\sigma}_{i: N}^{\mathrm{HW}}(t)=\sum_{j=i}^{N} w_{j}^{i: N}(0) \bar{\sigma}_{j}(t) .
$$

Write $w_{j}^{i: N}:=w_{j}^{i: N}(0)$ and make the convention that

$$
\bar{\sigma}_{i}(t)=\bar{\sigma}_{i: N}(t)=0 \quad \text { when } \quad t>T_{i},
$$

then an insightful presentation of equation (8) is:

$$
\begin{aligned}
& \bar{\sigma}_{1: N}^{\mathrm{HW}}(t)=w_{1}^{1: N} \bar{\sigma}_{1}(t)+\ldots+w_{N}^{1: N} \bar{\sigma}_{N}(t) \\
& \begin{array}{ccc}
\vdots & \ddots & \vdots \\
\bar{\sigma}_{N: N}^{\mathrm{HW}}(t)= & & w_{N}^{N: N} \bar{\sigma}_{N}(t)
\end{array}
\end{aligned}
$$

If $W$ is this upper triangular non-singular weight matrix (with upper triangular inverse $W^{-1}$ ) then these volatility vectors can be jointly related through the matrix equation

$$
\left[\bar{\sigma}_{\cdot: N}\right]=W[\bar{\sigma} .] .
$$

The swap rate volatility under relative perturbation (equation (3)) of the $k^{\text {th }}$ volatility is

$$
\left[\bar{\sigma}_{\cdot: N}\right] \rightarrow\left[\bar{\sigma}_{\cdot: N}\right]+\varepsilon\left[\begin{array}{lllllll}
0 & \ldots & 0 & \bar{\sigma}_{k: N} & 0 & \ldots & 0
\end{array}\right]^{\top}
$$

Note that the swap rate correlation is left unaltered. The corresponding perturbation in the BGM volatility vectors is given by

$$
[\bar{\sigma} .] \rightarrow[\bar{\sigma} .]+\varepsilon W^{-1}\left[\begin{array}{lllllll}
0 & \ldots & 0 & \bar{\sigma}_{k: N} & 0 & \ldots & 0
\end{array}\right]^{\top}
$$


Note that only the volatility vectors $\bar{\sigma}_{k}(t), \ldots, \bar{\sigma}_{N}(t)$ are affected (due to the upper triangular nature of $W^{-1}$ ), which are the vectors that underly $\bar{\sigma}_{k: N}(t)$ in the Hull and White approximation. With the new Libor volatility vectors, prices can be recomputed in the BGM model and the vegas calculated.

\section{$5 \quad$ Return to Re-Calibration Approach}

The tools developed in the previous sections will be used in this section to explain the vega results of the re-calibration approach of section 2 . To check whether the true swap rate dynamics are captured we simply have to verify that the swap rate volatility is perturbed as prescribed by equation (10). This test was performed for the THFRV deal setup of section 2. The results of this test are that for the re-calibration approach the swap rate volatility increment (in the limit) is completely different from the increment prescribed by equation (10). This holds for all buckets. For illustration we restrict to the exhibit in figure 5 .

Figure 5 displays the quadratic variation increment of the $30 \times 1$ swap rate $S_{30: 30}$ for purpose of calculating the swap vega corresponding to the $30 \times 1$ bucket. As can be seen from the figure, the distribution of the swap rate quadratic variation increment is concentrated on the begin and end time periods and is even negative for the second time period. This deviates away from the natural and intuitive evenly distribution as indicated as the correct approach.

An explanation of the results found in section 2 may now be given. From equation (2) it follows that the simulation variance of the vega is given by

$$
\begin{aligned}
\operatorname{Var}\left[\mathcal{V}_{i: N}^{100 \mathrm{bp}}\right] & =c^{2} \operatorname{Var}\left[P_{i: N}-P\right] \\
& =c^{2}\left\{\operatorname{Var}\left[P_{i: N}\right]-2 \operatorname{Cov}\left[P_{i: N}, P\right]+\operatorname{Var}[P]\right\}
\end{aligned}
$$

The vega standard error is thus minimized if the covariance between the discounted payoff in either the original and the perturbed model is largest. This occurs under small perturbations of volatility as implied by the constant volatility regime. In the presence of a perturbation such as the THFRV recalibration of figure 5 however the stochasticity in the simulation basically is moved around to other independent stochastic increments, thereby decreasing the covariance. This leads to a large uncertainty in the vega.

\section{$6 \quad$ Numerical Results}

In this section the algorithm of section 4 is applied to the deal setup of section

2. The results for a simulation with 10,000 scenarios have been displayed in 


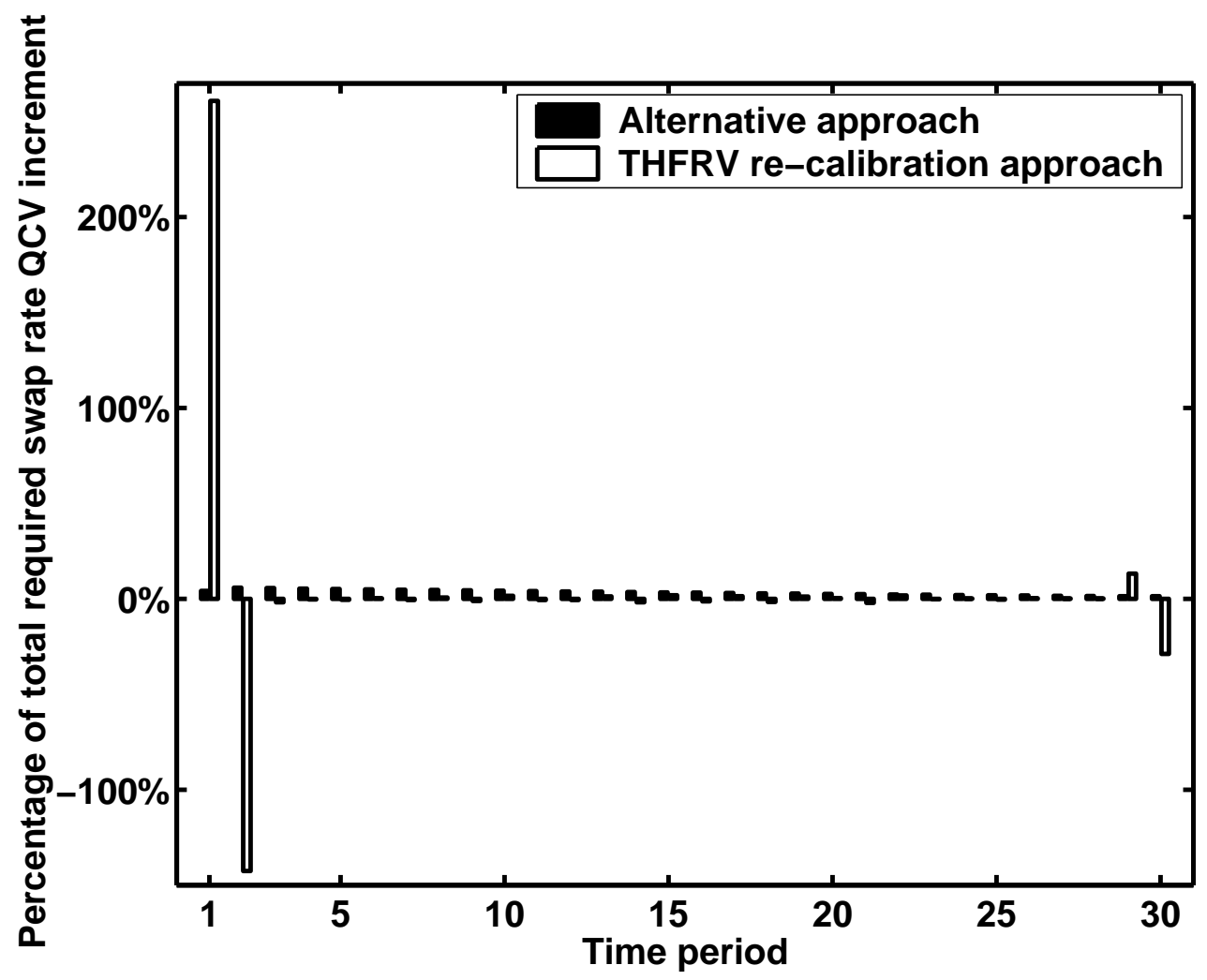

Figure 5: Swap rate volatility dynamics for both the approach of section 4 and the re-calibration approach in the THFRV deal setup of section 2 . Concern here is the calculation of swap vega corresponding to bucket 30 . To accomplish this, the price differential has to be computed in the limit of the $30 \times 1$ swaption implied volatility perturbation $\Delta \sigma$ tending to zero. This implies a swap rate quadratic variation increment of $30 \Delta \sigma^{2}$. This total variation increment has to be distributed over all time periods. The first data set displays the correct way of distributing the variation increment, namely proportional to the swap rate instantaneous volatility over the period. The second data set displays the distribution as implied by re-calibrating the THFRV model to only the swaption volatilities. Note that for each data set the sum of the variation increments equals $100 \%$. 
figure 6. Note that the approach yields slightly negative vegas for buckets 1730. The negativity can be explained by the results of appendix B. Namely for the analytically tractable setup of a two stock Bermudan option it is shown that negativity of vega can occasionally occur.

Remark 6 (Relative versus absolute shifting) The vegas have been displayed for the relative perturbation method. These vegas have been calculated as well using the absolute perturbation method. The differences in the vegas for the two methods are minimal; for any vega with absolute value above 1 bp the difference is less than $4 \%$, and for any vega with absolute value below $1 \mathrm{bp}$ the difference is always less than a third of a basis-point.

\section{Comparison with the Swap Market Model}

This section reports the results of an empirical comparison with the swap market model, which is the canonical model for computing swap vega per bucket. The key is to compare the Libor BGM model against a swap market model with the very same swap rate quadratic cross-variation structure. This (approximate) equivalence between the two models was established in [JoT02], equation (3.8).

Numerical Results. The test was performed for an 11NC1 pay-fixed Bermudan option on a swap with annual fixed and floating payments. A single-factor Libor BGM model was taken with constant volatility calibrated to the cap volatility curve of 10 October 2001. The zero rates were taken to be flat at 5\%. In the Monte Carlo simulation of the SMM we applied the discretization suggested in lemma 5 of [GlZ00]. Results may be found in table 2 and have been displayed partially in figures 7 and 8. In this particular case the BGM Libor model reproduces the swap vegas of the swap market model with high accuracy.

\section{Conclusions}

This article presented a novel approach for calculating swap vega per bucket in the Libor BGM model. We showed that for some forms of the volatility an approach based on re-calibration may lead to a large uncertainty in estimated swap vega, as the instantaneous volatility structure may be distorted by re-calibration. This does not happen in the case of constant swap rate volatility. We then derived an alternative approach, not based on recalibration, by comparison with the swap market model. The strength of the method is that it accurately estimates swaption vegas for any volatility function and at a low number of simulation paths. The key to the method 


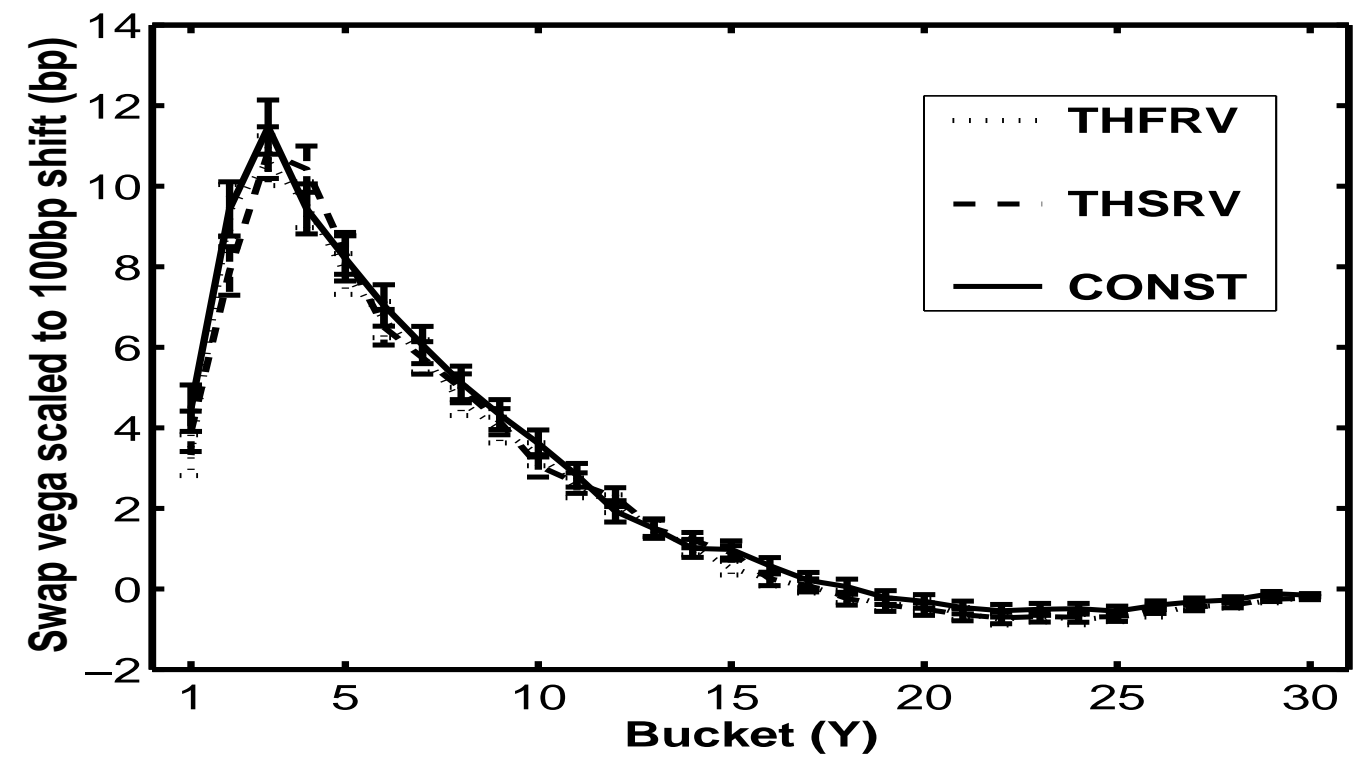

Figure 6: Method of section 4 swap vega results for 10,000 simulation paths. Error bars denote a $95 \%$ confidence bound based on the standard error. The vega is a scaled numerical derivative and we verified that it is insensitive to the actual size of the small volatility perturbation used (see also remark 3).

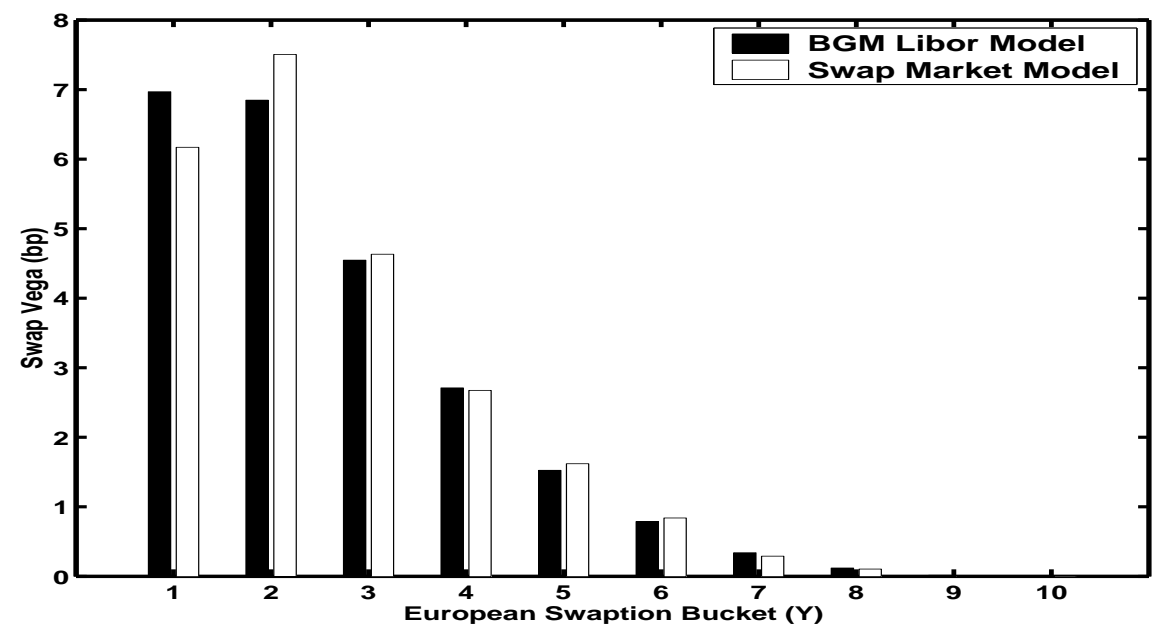

Figure 7: Comparison of LMM and SMM for swap vega per bucket. The fixed rate of the swap is $5 \%$. 
Table 2: Swap vega per bucket test results for varying strikes (fixed rate of the swap). Prices and vegas are stated in basis points. The standard error is denoted within parentheses. 10,000 simulation paths.

\begin{tabular}{|c|c|c|c|c|c|c|c|c|c|c|c|c|c|}
\hline $\begin{array}{c}\text { Fixed } \\
\text { Rate }\end{array}$ & $2 \%$ & $3 \%$ & $3.5 \%$ & $4 \%$ & $4.5 \%$ & $5 \%$ & $6 \%$ & $7 \%$ & $8 \%$ & $9 \%$ & $10 \%$ & $12 \%$ & $15 \%$ \\
\hline Value & $\begin{array}{r}2171 \\
(4)\end{array}$ & $\begin{array}{r}1476 \\
(5)\end{array}$ & $\begin{array}{r}1138 \\
(5)\end{array}$ & $\begin{array}{r}829 \\
(5)\end{array}$ & $\begin{array}{r}585 \\
(5)\end{array}$ & $\begin{array}{r}410 \\
(4)\end{array}$ & $\begin{array}{r}210 \\
(3)\end{array}$ & $\begin{array}{r}112 \\
(2)\end{array}$ & $\begin{array}{c}64 \\
(2)\end{array}$ & $\begin{array}{r}36 \\
(1)\end{array}$ & $\begin{array}{r}21 \\
(1)\end{array}$ & $\begin{array}{r}8 \\
(1)\end{array}$ & $\begin{array}{r}2 \\
(0)\end{array}$ \\
\hline $1 \mathrm{Y}$ & -2.0 & -2.0 & 2.6 & 10.9 & 11.1 & 7.0 & 1.2 & 0.1 & 0.0 & 0.0 & 0.0 & 0.0 & 0.0 \\
\hline $2 \mathrm{Y}$ & 1.5 & 1.6 & 1.0 & 2.6 & 5.7 & 6.8 & 4.0 & 1.0 & 0.0 & 0.0 & 0.0 & 0.0 & 0.0 \\
\hline $3 \mathrm{Y}$ & 0.0 & 0.0 & -0.3 & 0.1 & 2.5 & 4.5 & 4.1 & 2.1 & 1.0 & 0.3 & 0.0 & 0.0 & 0.0 \\
\hline $4 Y$ & 0.0 & 0.0 & -0.1 & -0.1 & 1.1 & 2.7 & 4.4 & 3.6 & 2.0 & 1.1 & 0.5 & 0.2 & 0.1 \\
\hline $5 \mathrm{Y}$ & 0.0 & 0.0 & -0.1 & -0.2 & 0.4 & 1.5 & 3.7 & 3.6 & 2.7 & 1.5 & 1.0 & 0.3 & 0.1 \\
\hline $6 \mathrm{Y}$ & 0.0 & 0.0 & -0.1 & -0.2 & 0.1 & 0.8 & 2.1 & 2.5 & 2.0 & 1.7 & 1.2 & 0.3 & 0.2 \\
\hline $7 \mathrm{Y}$ & 0.0 & 0.0 & -0.1 & -0.2 & 0.0 & 0.3 & 1.3 & 1.8 & 1.8 & 1.6 & 1.1 & 0.5 & 0.0 \\
\hline $8 Y$ & 0.0 & 0.0 & 0.0 & -0.1 & -0.1 & 0.1 & 0.7 & 1.3 & 1.5 & 1.3 & 1.3 & 0.9 & 0.3 \\
\hline $9 \mathrm{Y}$ & 0.0 & 0.0 & 0.0 & -0.1 & -0.1 & 0.0 & 0.3 & 0.7 & 0.8 & 0.8 & 0.8 & 0.6 & 0.3 \\
\hline $10 \mathrm{Y}$ & 0.0 & 0.0 & 0.0 & 0.0 & 0.0 & 0.0 & 0.1 & 0.3 & 0.3 & 0.4 & 0.4 & 0.3 & 0.2 \\
\hline $\begin{array}{l}\text { Total } \\
\text { Vega }\end{array}$ & -0.5 & -0.4 & 2.9 & 12.8 & 20.8 & 23.8 & 21.9 & 16.9 & 12.3 & 8.8 & 6.2 & 3.1 & 1.0 \\
\hline
\end{tabular}

\section{SWAP MARKET MODEL}

\begin{tabular}{|c|c|c|c|c|c|c|c|c|c|c|c|c|c|}
\hline $\begin{array}{r}\text { Fixed } \\
\text { Rate }\end{array}$ & $2 \%$ & $3 \%$ & $3.5 \%$ & $4 \%$ & $4.5 \%$ & $5 \%$ & $6 \%$ & $7 \%$ & $8 \%$ & $9 \%$ & $10 \%$ & $12 \%$ & $15 \%$ \\
\hline Value & $\begin{array}{r}2172 \\
(6)\end{array}$ & $\begin{array}{r}1480 \\
(6)\end{array}$ & $\begin{array}{r}1146 \\
(6)\end{array}$ & $\begin{array}{r}841 \\
(5)\end{array}$ & $\begin{array}{r}592 \\
(5)\end{array}$ & $\begin{array}{r}411 \\
(4)\end{array}$ & $\begin{array}{r}204 \\
(4)\end{array}$ & $\begin{array}{r}109 \\
(3)\end{array}$ & $\begin{array}{r}61 \\
(2)\end{array}$ & $\begin{array}{r}34 \\
(1)\end{array}$ & $\begin{array}{r}19 \\
(1)\end{array}$ & $\begin{array}{r}7 \\
(1)\end{array}$ & $\begin{array}{r}1 \\
(0)\end{array}$ \\
\hline $1 Y$ & -1.9 & -0.7 & 4.4 & 11.3 & 11.5 & 6.2 & 0.4 & 0.0 & 0.0 & 0.0 & 0.0 & 0.0 & 0.0 \\
\hline $2 \mathrm{Y}$ & 1.6 & 1.6 & 1.1 & 2.2 & 5.2 & 7.5 & 3.6 & 0.5 & 0.0 & 0.0 & 0.0 & 0.0 & 0.0 \\
\hline $3 Y$ & 0.0 & -0.1 & -0.4 & 0.0 & 2.0 & 4.6 & 4.7 & 2.2 & 0.6 & 0.2 & 0.0 & 0.0 & 0.0 \\
\hline $4 Y$ & 0.0 & -0.1 & -0.2 & -0.1 & 0.9 & 2.7 & 4.8 & 3.7 & 1.7 & 0.8 & 0.3 & 0.1 & 0.0 \\
\hline $5 \mathrm{Y}$ & 0.0 & 0.0 & -0.2 & -0.2 & 0.4 & 1.6 & 3.7 & 3.0 & 2.3 & 1.2 & 0.5 & 0.1 & 0.0 \\
\hline $6 Y$ & 0.0 & 0.0 & -0.1 & -0.2 & 0.1 & 0.8 & 2.6 & 3.3 & 3.1 & 2.3 & 1.2 & 0.2 & 0.0 \\
\hline $7 \mathrm{Y}$ & 0.0 & 0.0 & -0.1 & -0.2 & -0.1 & 0.3 & 1.3 & 2.0 & 1.9 & 1.3 & 1.4 & 0.8 & 0.1 \\
\hline $8 Y$ & 0.0 & 0.0 & 0.0 & -0.1 & -0.1 & 0.1 & 0.8 & 1.3 & 1.5 & 1.5 & 1.2 & 0.6 & 0.2 \\
\hline $9 \mathrm{Y}$ & 0.0 & 0.0 & 0.0 & -0.1 & -0.1 & 0.0 & 0.4 & 0.9 & 1.0 & 1.0 & 0.9 & 0.7 & 0.3 \\
\hline $10 \mathrm{Y}$ & 0.0 & 0.0 & 0.0 & 0.0 & 0.0 & 0.0 & 0.1 & 0.3 & 0.4 & 0.5 & 0.5 & 0.4 & 0.3 \\
\hline $\begin{array}{l}\text { Total } \\
\text { Vega }\end{array}$ & -0.3 & 0.6 & 4.5 & 12.6 & 19.9 & 23.8 & 22.3 & 17.2 & 12.5 & 8.8 & 6.0 & 2.9 & 0.9 \\
\hline
\end{tabular}




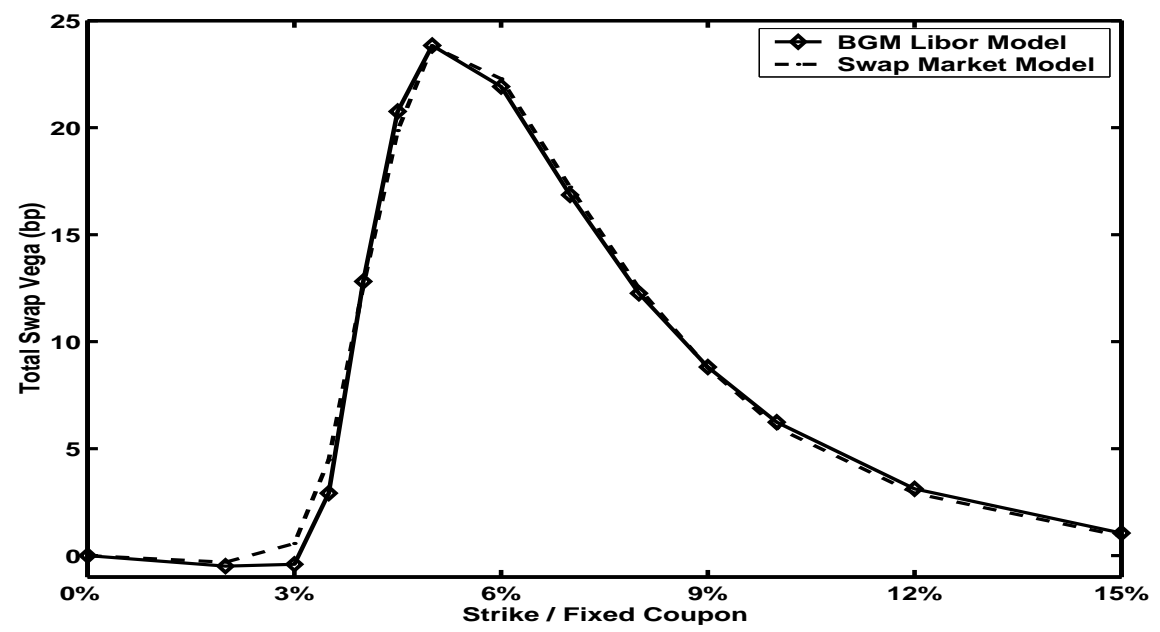

Figure 8: Comparison of LMM and SMM for total swap vega against strike.

is that the perturbation in the Libor volatility is distributed in a clear, stable and well understood fashion, whereas in the re-calibration method the change in volatility is hidden and potentially unstable. We also showed for a Bermudan swaption deal that our method yields similar swap vega as found in a swap market model. 


\section{References}

[BiD77] Bickel, P.J., Doksum, K.J.: Mathematical Statistics: Basic Ideas and Selected Topics. Englewood Cliffs, NJ: Prentice Hall (1977)

[BDB98] Brace, A., Dunn, T., Barton, G.: Towards a Central Interest Rate Model. Paris: ICBI Global Derivatives Conference (1998)

[BGM97] Brace, A., Gạtarek, D., Musiela, M.: The Market Model of Interest Rate Dynamics. Mathematical Finance 7 (2) 127-155 (1997)

[D'A02] D'Aspremont, A.: Calibration and Risk-Management Methods for the Libor Market Model Using Semidefinite Programming. Paris: Ph.D. Thesis Ecole Polytechnique (2002)

[G1Z99] Glasserman, P., Zhao, X.: Fast Greeks by Simulation in Forward LIBOR Models. Journal of Computational Finance 3 (1) 5-39 (1999)

[GlZ00] Glasserman, P., Zhao, X.: Arbitrage-free Discretization of Lognormal Forward Libor and Swap Rate Models. Finance and Stochastics 4 35-68 (2000)

[Hull00] Hull, J.C.: Options, Futures and Other Derivatives. $4^{\text {th }}$ edition. Englewood Cliffs, NJ: Prentice Hall (2000)

[HuW00] Hull, J.C., White, A.: Forward Rate Volatilities, Swap Rate Volatilities, and Implementation of the LIBOR Market Model. Journal of Fixed Income 3 46-62 (2000)

[Jam96] Jamshidian, F.: LIBOR and Swap Market Models and Measures. London: Sakura Global Capital Working Paper (1996)

[Jam97] Jamshidian, F.: LIBOR and Swap Market Models and Measures. Finance and Stochastics 1 293-330 (1997)

[JoT02] Joshi, M.S., Theis, J.: Bounding Bermudan Swaptions in a Swap-Rate Market Model. Quantitative Finance 2 370-377 (2002)

[LoS01] Longstaff, F.A., Schwartz, E.S.: Valuing American Options by Simulation: A Simple Least-squares Approach. The Review of Financial Studies 14 (1) 113-147 (2001)

[MSS97] Miltersen, K.R., Sandmann, K., Sondermann, D.: Closed Form Solutions for Term Structure Derivatives with Log-normal Interest Rates. Journal of Finance 52 (1) 409-430 (1997)

[Reb98] Rebonato, R.: Interest Rate Option Models. $2^{\text {nd }}$ edition. Chichester: J. Wiley \& Sons (1998) 
[Reb01] Rebonato, R.: Accurate and Optimal Calibration to Co-Terminal European Swaptions in a FRA-based BGM Framework. London: Royal Bank of Scotland Working Paper (2001)

[Reb02] Rebonato, R.: Modern Pricing of Interest-Rate Derivatives. New Jersey: Princeton University Press (2002) 


\section{Appendix A: Two Stage Bootstrap}

In this appendix the two stage bootstrap method is outlined for calibrating the Libor BGM model to swaption volatility. It is presented here for illustration of the vega calculation method only.

The idea is described as follows. It is assumed that the forward rate correlation matrix is given. The method consists of two stages. In the first stage, the swap rate volatility is assumed piece-wise constant satisfying the time-homogeneity restriction. The swap rate instantaneous volatility may then be calculated by means of a bootstrap. In the second stage, the forward rate volatility is determined to match the required swap rate instantaneous volatility found in the first stage.

The bootstrap of the first stage is well known in the literature and may for example be found in [HuW00], equation (18). The second stage may also be calculated by means of a bootstrap as shown below. Given a time period index $m$ spanning the period $\left[T_{m-1}, T_{m}\right]$ the absolute level of forward rate or swap rate instantaneous volatility over that period is denoted by $\sigma_{i}(m)$ or $\sigma_{i: N}(m)$, respectively. The forward rate instantaneous volatility for period $m$ is determined iteratively from rate $N$ down to $m$. From equation (8) it follows

$$
\sigma_{i: N}(m)^{2}=\sum_{j=i}^{N} \sum_{k=i}^{N} w_{j}^{i: N} w_{k}^{i: N} \sigma_{j}(m) \sigma_{k}(m) \rho_{j k}(m) .
$$

Note that $\sigma_{i}(m)$ is the only unknown variable in this equation, the terms $\sigma_{j}(m), j=i+1, \ldots, N$, have already been determined in the previous iteration. Therefore $\sigma_{i}(m)$ solves the quadratic equation

$$
\alpha \sigma_{i}(m)^{2}+\beta \sigma_{i}(m)+\gamma=0,
$$

with

$$
\begin{aligned}
\alpha & :=\left(w_{i}^{i: N}\right)^{2} \\
\beta & :=2 \sum_{j=i+1}^{N} w_{j}^{i: N} w_{i}^{i: N} \sigma_{j}(m) \rho_{i j}(m) \\
\gamma & :=-\sigma_{i: N}(m)^{2}+\sum_{j=i+1}^{N} \sum_{k=i+1}^{N} w_{j}^{i: N} w_{k}^{i: N} \sigma_{j}(m) \sigma_{k}(m) \rho_{j k}(m) .
\end{aligned}
$$

The relevant root is given by

$$
\sigma_{i}(m)=\frac{-\beta+\sqrt{\beta^{2}-4 \alpha \gamma}}{2 \alpha} .
$$


The other root would yield non-positive absolute levels of volatility since $\alpha>0$ and $\beta \geq 0$. The latter inequality holds whenever the correlation is positive, which is not an unrealistic assumption for interest rates.

\section{Appendix B: Negative vega for a two stock Bermudan option}

In this appendix a two stock Bermudan option is studied; in particular its vega per bucket is analyzed and it is shown that it is negative for certain situations. The holder of a two stock Bermudan has the right to call the first stock $S_{1}$ at strike $K_{1}$ at time $T_{1}$; if he or she decides to hold on to the option then the right remains to call the second stock $S_{2}$ at strike $K_{2}$ at time $T_{2}$; if this right is not exercised then the option becomes worthless. Here $T_{1}<T_{2}$.

Model setup. The Bermudan option will be valued in the standard BlackScholes world. Under the risk-neutral measure the stock prices satisfy the following SDEs

$$
\begin{gathered}
\frac{\mathrm{d} S_{i}}{S_{i}}=r \mathrm{~d} t+\sigma_{i} \mathrm{~d} W_{i}, \quad i=1,2, \\
\mathrm{~d} W_{1} \mathrm{~d} W_{2}=\rho \mathrm{d} t .
\end{gathered}
$$

Here $\sigma_{i}$ is the volatility of the $i^{\text {th }}$ stock. $W_{i}, i=1,2$, are Brownian motions under the risk-neutral measure, with correlation $\rho$. It follows that the time- $T_{1}$ stock prices are distributed as follows

$$
S_{i}\left(T_{1}\right)=F\left(S_{i}(0), 0 ; T_{1}\right) \exp \left\{\sigma_{i} \sqrt{T_{1}} Z_{i}-\frac{1}{2} \sigma_{i}^{2} T_{1}\right\}, \quad i=1,2,
$$

where the pair $\left(Z_{1}, Z_{2}\right)$ is standard bivariate normally distributed with correlation $\rho$ and where

$$
F(S, t ; T):=S \exp \{r(T-t)\}
$$

is the time- $t$ forward price for delivery at time $T$ of a stock with current price $S$. At time $T_{1}$ the holder of the Bermudan will choose whichever of the two following alternatives has a higher value: either calling the first stock or holding onto the option on the second stock; the value of the latter is given by the Black-Scholes formula. Therefore the (cash-settled) payoff $V\left(S_{1}\left(T_{1}\right), S_{2}\left(T_{1}\right), T_{1}\right)$ of the Bermudan at time $T_{1}$ is given by

$$
\max \left\{\left(S_{1}\left(T_{1}\right)-K_{1}\right)_{+}, \mathrm{BS}_{2}\left(S_{2}\left(T_{1}\right), T_{1}\right)\right\},
$$

where BS is the Black-Scholes formula,

$$
\begin{aligned}
\mathrm{BS}_{i}(S, T) & =e^{-r\left(T_{i}-T\right)}\left\{F\left(S, T ; T_{i}\right) N\left(d_{1}^{(i)}\right)-K_{i} N\left(d_{2}^{(i)}\right)\right\} \\
d_{1,2}^{(i)}(S, T) & =\frac{\ln \left(F\left(S, T ; T_{i}\right) / K_{i}\right) \pm \frac{1}{2} \sigma_{i}^{2} T}{\sigma_{i} \sqrt{T}} .
\end{aligned}
$$


Table 3: Deal setup for examples where a vega per bucket for the two stock Bermudan option is negative.

\begin{tabular}{lcr}
\hline spot price for stock 1 & $S_{1}(0)$ & 150 \\
spot price for stock 2 & $S_{2}(0)$ & 140 \\
strike price for stock 1 & $K_{1}$ & 100 \\
strike price for stock 2 & $K_{2}$ & 100 \\
exercise time for stock 1 & $T_{1}$ & $1 \mathrm{Y}$ \\
exercise time for stock 2 & $T_{2}$ & $2 \mathrm{Y}$ \\
volatilities & $\sigma_{i}$ & Variable \\
correlation & $\rho$ & 0.9 \\
risk-free rate & $\mathrm{r}$ & $5 \%$ \\
\hline
\end{tabular}

Here $N(\cdot)$ is the cumulative normal distribution function. The time-zero value $V\left(S_{1}, S_{2}, 0\right)$ of the Bermudan option may thus be computed by a bivariate normal integration of the discounted version of the above payoff

$$
V\left(S_{1}, S_{2}, 0\right)=e^{-r T_{1}} \mathbb{E}\left[V\left(T_{1}, S_{1}\left(T_{1}\right), S_{2}\left(T_{1}\right)\right)\right]
$$

Vega per bucket. The vega per bucket $\mathcal{V}_{i}$ is defined as

$$
\mathcal{V}_{i}:=\frac{\partial V\left(S_{1}, S_{2}, 0\right)}{\partial \sigma_{i}}, \quad i=1,2
$$

The vega may be numerically approximated by finite differences

$$
\mathcal{V}_{i}=\frac{V\left(S_{1}, S_{2}, 0 ; \sigma_{i}+\Delta \sigma_{i}\right)-V\left(S_{1}, S_{2}, 0 ; \sigma_{i}\right)}{\Delta \sigma_{i}}+\mathcal{O}\left(\Delta \sigma_{i}^{2}\right), \quad i=1,2,
$$

for a small volatility perturbation $\Delta \sigma_{i} \ll 1$. We claim that the vega per bucket may possibly be negative; this may occur for both the first and the second bucket. To provide examples of vega negativity, the vega per bucket has been computed for the deal setup described in table 3. Results have been displayed in table 4 . The volatility was perturbed by a small amount. It was verified that the resulting vega was insensitive to either the perturbation size or the density of the $2 \mathrm{D}$ integration grid. The results clearly establish instances where a vega per bucket is negative, both for the first and second bucket. To ensure that the negative vega was not due to an implementation error, an alternative valuation of the two stock Bermudan option was developed in a private paper of the authors, available from the authors upon 
Table 4: Examples where a vega per bucket for the two stock Bermudan option is negative. The vega has been re-scaled to a 100 bp volatility shift $(1 \%), \mathcal{V}_{i}^{100 \mathrm{bp}}=(0.01) \mathcal{V}_{i}$

\begin{tabular}{lrrrrr}
\hline & $\sigma_{1}$ & $\sigma_{2}$ & price & $\mathcal{V}_{1}^{100 \mathrm{bp}}$ & $\mathcal{V}_{2}^{100 \mathrm{bp}}$ \\
\hline scenario 1 & $10 \%$ & $30 \%$ & 64.53 & -0.45 & 0.56 \\
scenario 2 & $30 \%$ & $10 \%$ & 65.11 & 0.56 & -0.44 \\
\hline
\end{tabular}

request. The alternative valuation is based on conditioning and involves a one-dimensional numerical integration over the Black formula. Because the alternative valuation method is different in nature, it may be viewed as an independent implementation of the two dimensional numerical integration. Indeed, the alternative independently implemented method yielded the exact same results. Moreover, we provide in the developments below an economic explanation for the occurrence of negative vega.

Economic explanation. Note in table 4 that the negative vegas occur in case of high correlation and for the bucket with lowest volatility. In the case of high correlation and one stock having significantly higher volatility than the other, we contend that the only added value of the additional option on the low volatility stock lies in offering protection against a down move of both stocks (recall that the stocks are highly correlated). Namely, consider the following two scenarios:

- Up move. Both stocks move up. Because the high volatility stock moves up much more than the low volatility stock, the high volatility call will be exercised.

- Down move. Both stocks move down. Because the high volatility stock moves down much more than the low volatility stock, the high volatility call becomes out of the money and the low volatility call will be exercised.

If now the volatility of the low volatility stock is increased by a small amount, then in the above scenarios the exercise strategy remains unchanged. Also, in case of an up move, the payoff remains unaltered. However, in case of a down move, the low volatility stock (volatility slightly increased) moves down more than in the unperturbed case. Therefore the payoff of the protection call decreases. In total, the value of the Bermudan option thus decreases. 